\title{
0 futuro do detalhamento arquitetônico: concepção e representação na era digital, e impactos para a formação dos arquitetos.
}

\section{Caio Magalhães Castriotto \\ Arquiteto e Urbanista Mestrando em Arquitetura, Tecnologia e Cidade, Unicamp caio.castriotto@gmail.com \\ Guilherme Giantini da Silva Carvalho}

Arquiteto e Urbanista Mestrando em Arquitetura, Tecnologia e Cidade, Unicamp giantinigui@gmail.com

\section{Verley Henry Côco Júnior}

Graduando em Arquitetura e Urbanismo, Unicamp verleyjr@gmail.com

\section{Gabriela Celani}

Arquiteta e Urbanista, Professora Titular da Faculdade de Engenharia Civil, Arquitetura e Urbanismo, Unicamp celani@fec.unicamp.br

\section{RESUMO:}

Este artigo tem como objetivo contribuir para a discussão sobre o ensino do detalhamento na formação de arquitetos no Brasil. Ele começa apresentando uma breve revisão da literatura sobre o detalhamento dos pontos de vista técnico e teórico, seguida por uma apresentação de exemplos recentes do uso de tecnologias de fabricação digital na arquitetura. É apresentada também uma descrição sobre a representação digital de detalhes arquitetônicos por meio de modelagem da informação do edifício e por representações algorítmicas e paramétricas. 0 artigo termina com uma discussão a respeito da relação entre o detalhe e o uso dos meios digitais no processo de projeto e produção da arquitetura, e suas implicações para a formação do arquiteto no século XXI.

Palavras-chave: Detalhe arquitetônico; métodos digitais; fabricação digital; zero tolerance; Level of Development

\section{ABSTRACT:}

This paper aims to contribute to the discussion about the teaching of detailing in the training of architects in Brazil. It begins by presenting a brief review of the literature on detailing from the technical and theoretical points of view, followed by a presentation of recent examples of the use of digital fabrication technologies in architecture. A description of the digital representation of architectural details is presented, through building information modeling and algorithmic and parametric representations. The article concludes with a discussion about the relationship between detailing and the use of digital media in the process of designing and producing architecture, and its implications for the training of the architect in the 21st century.

Palavras-chave: Architectural detail; digital methods; digital fabrication; zero tolerance; Level of Development. 


\section{INTRODUÇÃ̃o}

Os prédios de Niemeyer são sempre interessantes e ousados na sua concepção, mas ele parece dar pouca atenção aos detalhes, o que acaba comprometendo a qualidade dos edifícios. (GROPIUS, W. In: XAVIER, A, 2003, p. 154.)

O detalhamento arquitetônico é essencial para a construção de um edifício com qualidade e fidelidade às intenções do projeto, segundo Allen e Rand (2007). Mas além de estar relacionado às questões de qualidade e de construtibilidade, o detalhe é o que distingue uma obra de arquitetura de uma simples construção civil, conferindo-lhe significado e expressão simbólica e sendo, portanto, uma etapa fundamental no processo de projeto (FORD, 2011).

No Brasil, o detalhamento arquitetônico costuma ser considerado uma etapa final do processo de projeto. Uma evidência disso é o fato de que na norma ABNT - NBR 6492 (1994) sobre "Representação de projetos de arquitetura", o detalhamento aparece apenas como "documento típico" da etapa de Projeto Executivo. De maneira semelhante, no "Roteiro para desenvolvimento de projetos de arquitetura da edificação" disponibilizado pelo Instituto dos Arquitetos do Brasil (IAB), a palavra detalhe aparece unicamente na fase do Projeto de execução, sendo anterior apenas à fase de Execução da obra. Seu papel seria de desenvolver e complementar as informações contidas nas plantas, cortes e fachadas.

Ainda segundo o documento do IAB,

[...] conforme o grau de industrialização dos componentes, os detalhes podem ser executivos ou esquemáticos. Neste último caso, os detalhes executivos são elaborados pelo fabricante do componente e aprovados pelo arquiteto; por exemplo: esquadrias de alumínio e forros industrializados. (IAB, p. 7)

O trecho acima afirma que o detalhe executivo seria necessário apenas na construção convencional. Ao se utilizar componentes industrializados, o detalhe esquemático bastaria. De fato, quando se prevê o uso de componentes industrializados em massa, as características dimensionais e as conexões entre esses elementos são definidas pelo fabricante. Nesse caso, os detalhes resultam apenas das diferentes possibilidades de combinação dos itens de catálogo.

Conforme Celani e Urano (2016), o surgimento da personalização em massa por meio do uso dos equipamentos industriais de controle numérico faz com que o detalhamento arquitetônico adquira novas características. Se, por um lado, Oxman e Oxman (2010) indicam que ele se assemelha ao detalhe da construção tradicional por sua singularidade, por outro ele depende de uma especificação ainda mais precisa, a qual está sujeita ao conhecimento das potencialidades dos equipamentos e dos materiais por parte do projetista. Embora o Brasil tenha baixos índices de industrialização do setor da construção civil quando comparado à Europa e Estados Unidos, autores como Vasconcelos (2002) e Silva (2009) afirmam que já existe uma disponibilidade de equipamentos de controle numérico no país, prontos para serem utilizados 
na produção de componentes construtivos customizados em massa. No entanto, os autores reconhecem o desconhecimento, por parte da maioria dos arquitetos brasileiros, sobre a existência, potencialidades e custo dessa nova forma de produção industrial.

No que se refere ao ensino, o detalhamento arquitetônico não parece receber a devida importância no Brasil. Ao consultar a grade curricular dos cursos elencados entre os melhores do Brasil, de acordo com a Folha de São Paulo (2016), constata-se que dos dez programas de graduação mais bem avaliados, apenas o da Universidade Federal do Rio de Janeiro (UFRJ) apresenta disciplinas direcionadas ao detalhamento, oferecidas como eletivas, ensinado a partir de temas específicos, como telhados, esquadrias, instalações sanitárias e elementos especiais. 0 fato de serem disciplinas eletivas pode indicar a menor prioridade no programa de ensino, caso não tenham sido recentemente implementadas, ou ainda não tenha ocorrido uma revisão curricular do curso a respeito deste assunto.

Os melhores programas internacionais de formação de arquitetos e urbanistas, elencados por rankings, como o QS World University Rankings by Subject (2016), apresentam um panorama mais homogêneo quanto ao ensino do detalhamento. Neles, o aprofundamento teórico e prático do assunto ocorre durante toda a produção acadêmica dos estudantes, através das disciplinas de projeto. Um programa que exemplifica tal abordagem integrada de ensino é o praticado pela Architectural Association School of Architecture, onde uma das atividades descritas na ementa curricular do quarto ano é o desenvolvimento de projetos de tipologias cívicas, culturais, comerciais ou industriais, desde a escala urbana até a escala do detalhe. Outro exemplo é o oferecido pela Graduate School of Design, da Universidade de Harvard. Na disciplina Innovative Construction in Japan, aborda-se a produção do detalhe a partir da arquitetura moderna japonesa como método de ensino e exploração da relação entre teorias de projeto e técnicas de construção.

A partir das constatações apresentadas acima e com o objetivo final de contribuir para a discussão sobre o ensino do detalhamento na formação de arquitetos no Brasil, este artigo apresenta uma revisão da literatura e reflexão a respeito da relação entre o detalhe e o uso dos meios digitais no processo de projeto e produção da arquitetura, sob os pontos de vista técnico e teórico.

\section{TECNOLOGIA DO DETALHAMENTO ARQUITETÔNICO}

São poucos os livros redigidos no Brasil sobre tecnologia do detalhamento arquitetônico. Os livros existentes sobre esse assunto enfocam, em geral, os aspectos de representação gráfica do detalhe, sem apresentar uma conceituação aprofundada dos pontos de vista técnico ou teórico, como exemplo, é possível citar o livro "Detalhes em Arquitetura", organizado pela Asbea (Associação Brasileira dos Escritórios de Arquitetura), onde desenhos de projeto executivo e de detalhamento arquitetônico de 48 escritórios são 
compilados ou "Detalhando a Arquitetura" de Antonio Ferreira Costa, publicado em 1997.

Um dos autores mais populares no Brasil na área de detalhamento arquitetônico é o americano Francis Ching, professor emérito da Universidade de Washington em Seattle, e autor de diversos livros desde a década de 1970, como Building Construction Illustrated e Architectural Graphics, que foram traduzidos para o português como "Técnicas de Construção Ilustradas e Desenho para Arquitetos". No primeiro, a categorização das técnicas de construção baseia-se nos elementos construtivos, como fundações, pisos, paredes, telhados, isolamento térmico e de umidade e portas e janelas.

O livro de Allen e Rand, Architectural Detailing, foi publicado originalmente nos Estados Unidos, em 1993, e posteriormente ampliado em edições subsequentes, sendo a última de 2016. Essa obra, mais voltadas efetivamente à categorização das técnicas de detalhamento, apresenta os detalhes de acordo com o tipo de problema a ser enfrentado, como controle dos fluxos de água, ar, calor, vapor e som, acomodação de movimentos, apoio estrutural, passagem de instalações e facilidade de montagem.

$\mathrm{Na}$ Europa, uma das obras mais completas sobre sistemas construtivos e detalhamento arquitetônico é o livro Constructing Architecture, organizado por Andrea Deplazes e publicado originalmente em 2005 em alemão. Ele possui sessões sobre materiais (alvenaria, concreto, madeira, etc.), elementos construtivos, componentes e estruturas, além de uma sessão sobre desempenho, na qual são apresentados conceitos e princípios, como a circulação do calor e a difusão da umidade na construção.

Embora as obras de Ching sejam visualmente muito bonitas e a de Allen e Rand possua uma categorização extremamente didática dos princípios do detalhamento, dada a época de suas versões originais, esses livros não chegam a levar em consideração o uso de máquinas de controle numérico, baseando-se nos princípios de produção industrializada em massa. Já a obra de Deplazes, mais recente, embora não apresente aspectos específicos sobre os detalhes produzidos para a fabricação digital, menciona essa possibilidade, em especial para o tratamento da madeira. 0 autor cita, inclusive, a perspectiva de viabilização econômica da produção industrial personalizada, mas sua ênfase ainda é na produção industrial em massa:

\footnotetext{
[...] the subsequent processing of the semi-finished products, the "refining" and the further processing towards prefabrication for building works, gives rise to an astounding suppleness in the material, allowing every shaping intervention - the CNC-controlled milling cutter, the robot machining - virtually without resistance. The term "modeling" is certainly apt here because not only complex patterns but also plastic shaping such as profiling and even threedimensional workpieces are produced whose surface developments can be defined numerically before machining [...] in the production line from the architects CAD system to the CAM and $\mathrm{CNC}$ roboting of the fabricator it is certainly realistic to order a "one-off" copy of a highly complicated carpentry joint, e.g. from a
} 
Japanese Shinto shrine, even for a relatively moderate price. (DEPLAZES, A, 2005, p.78)

Aspectos específicos sobre detalhes de produtos fabricados digitalmente podem ser encontrados em livros que não são normalmente consultados por arquitetos. A obra Product Design for Manufacture and Assembly de Boothroyd, Dewhurst e Knight (2002), por exemplo, que faz parte da literatura fundamental para a formação do engenheiro mecânico, apresenta conceitos sobre a seleção de materiais e de processo de fabricação, as características desejáveis da geometria dos objetos visando sua produção pelos diferentes processos industriais e sua montagem - manual e robotizada - entre outros assuntos. Obviamente, um livro como esse não se preocupa com questões estéticas ou estilísticas, mas a transposição de seus conceitos para o campo do detalhamento arquitetônico poderia tornar os projetos muito mais efetivos e economicamente viáveis.

\section{TEORIA DO DETALHAMENTO ARQUITETÔNICO}

Edward R. Ford, professor da Universidade da Virgínia, é provavelmente o pesquisador que possui o maior número de obras publicadas recentemente sobre o detalhamento arquitetônico sob o ponto de vista teórico. Ele é autor dos livros The Details of Modern Architecture (MIT Press, 1990), The Details of Modern Architecture vol. 2: 1928 to 1988 (MIT Press, 1996), Five Houses, Ten Details (Princeton Architectural Press, 2009) e The Architectural Detail (Princeton Architectural Press, 2011).

Ford (2011) propõe uma classificação do detalhe arquitetônico de acordo com suas características, intenções e razões, em cinco categorias: detalhe como abstração, detalhe como tema, detalhe como atividade subversiva, detalhe como representação da estrutura e detalhe como articulação. 0 detalhe como abstração pode ser caracterizado como "nãodetalhe" e é manifestado na maioria das obras modernistas. Trata-se da omissão de elementos de pequena escala, de conexões e de outros aspectos técnicos do edifício, visando eliminar qualquer elemento que possa ser lido como um ornamento:

\footnotetext{
Detalhar é eliminar não apenas "molduras desnecessárias", mas também elementos em pequena escala que evidenciam o fato de que um problema técnico foi solucionado: como a água é desviada do topo de uma parede ou de um peitoril, como um pano de vidro é fixado, ou como dois materiais são unidos. Na arquitetura tradicional, havia elementos visíveis para resolver essas tarefas: cimalhas, peitoris, pinázios, molduras. Na prática do não-detalhe esses elementos, na maioria das vezes, estão presentes, mas não são perceptíveis. (FORD, 2011.p. 21)
}

Essa concepção do detalhe arquitetônico tem suas origens no manifesto de Loos (1908) e no repúdio aos exageros do ecletismo do século XIX. O detalhe como tema, por sua vez, é apresentado em oposição ao "nãodetalhe". Nessa categoria, os detalhes, de característica mais ornamental, estariam repetidos nas partes e no todo do edifício, em diferentes escalas. Segundo o autor, a origem desse tipo de detalhe estaria no gótico, mas ele 
estaria presente até mesmo no modernismo "[...] sob diferentes disfarces e fundamentos filosóficos" (FORD, 2011, p. 28). Um exemplo seriam as textile houses de Frank Lloyd Wright, nos anos 1920. O detalhe como atividade subversiva, também de caráter ornamental, é caracterizado por elementos desvinculados do contexto restante da obra, apresentando suas próprias regras, configurações e expressão.

0 detalhe como representação da estrutura trata de sua expressão como ordem arquitetônica. Nesse caso, o elemento detalhado não precisaria ter necessariamente uma função estrutural, mas deveria representar, retoricamente, as questões tectônicas do edifício, com o intuito de estabelecer uma ordem de distanciamentos, efeitos visuais ou materialidade. Segundo o autor, apesar de condenarem o uso de elementos falsos, os arquitetos modernos muitas vezes recorreram a esta categoria de detalhe quando a exposição direta da estrutura era impossível. 0 exemplo mais famoso disto, inclusive citado por Ford (2011) é o canto do Alumni Memorial Hall de Mies Van der Rohe, no qual, por motivos de segurança contra incêndio, o pilar de aço foi revestido primeiramente em concreto e, externamente, em chapa de aço, dando uma aparência de estrutura exposta.

Finalmente, o detalhe como conexão entre as partes e materiais do edifício seria uma versão mais sincera da categoria anterior e, nesse sentido, estaria na essência da arquitetura, "[...] pois a maneira como se entende as partes de um edifício e as relações entre elas pode ser uma oportunidade para expressar grandes intenções em arquitetura" (FORD, 2011, p. 37). Além disso, "nossa compreensão da arquitetura depende [...] não apenas da percepção das partes, mas da percepção de que cargas são transferidas de uma a outra parte do edifício, que essas partes estão conectadas firmemente, e que estão equilibradas" (FORD, 2011, p. 306-307). Como exemplo para esse caso, o autor apresenta o projeto de Beurs van Berlage, para a Bolsa de Amsterdã, de 1903, no qual o arquiteto enfatiza a mudança de materiais entre a parede portante de tijolos e a estrutura metálica do telhado.

Após descrever a importância do tipo de detalhamento arquitetônico que ele denomina de conexão, Ford relativiza sua importância no cenário contemporâneo em que, segundo ele, "[...] a junta articulada caiu em desuso" (FORD, 2011, p. 41), em consequência da grande precisão dos equipamentos de fabricação digital. Kolarevic (2014), por outro lado, refuta essa ideia uma vez que, independentemente da precisão dos meios de produção digitais, as propriedades físicas de dilatação dos materiais continuarão exigindo a previsão de graus de tolerância que tornam necessário o desenvolvimento de detalhes na construção.

Mas, de acordo com Ford (2011), mesmo as teorias mais recentes não foram capazes, ainda, de responder de forma precisa qual o papel do detalhe na produção da arquitetura contemporânea com o uso dos meios digitais. Esse é justamente o tema do número especial da revista Architectural Detail editorada por Mark Garcia e intitulada Future Details of Architecture (2014). Essa publicação, organizada em resposta à afirmação de Greg Lynn de que o 
detalhe teria morrido, mostra, pelo contrário, que o detalhe arquitetônico estaria mais vivo e ativo do que nunca. 0 número inclui textos do próprio Ford, além de diversos outros arquitetos que mostram, a partir do uso de tecnologias digitais de projeto e de fabricação, que a atenção ao detalhe arquitetônico é um aspecto fundamental para a inovação na arquitetura. Ford (2014) conclui que o futuro do detalhe é, na realidade, o futuro da arquitetura.

Outra publicação da $\mathrm{AD}$ relacionada ao tema é o número especial intitulado High Definition: Zero Tolerance in Design and Production, organizado por Bob Sheil em 2014. Uma das principais características da fabricação digital é sua elevada precisão. Esse é um aspecto que deve ser levado em consideração ao se projetar um elemento construtivo para ser produzido por equipamentos de controle numérico. De acordo com Nolte e Witt (2014) em seu artigo para a $\mathrm{AD}$, a rápida evolução das ferramentas e equipamentos de Fabricação Digital permite gerar e simular diferentes alternativas praticamente em tempo real. Os modelos digitais são repositórios de informações não apenas geométricas, mas de material, regras e propriedades.

Em 2008, Peggy Deamer já apontava para a necessidade de se repensar o detalhe arquitetônico frente à mudança nos meios de produção do edifício: "the change to architectural production brought on by parametric design, though not normally linked to a discourse of detail, is in fact essentially tied to it and this is indeed a moment of architectural detail crisis" (DEAMER, 2010, p. 81). Segundo a autora, as condicionantes tecnológicas atuais desqualificariam as razões de Loos para criminalizar o ornamento:

[...] contemporary practice revitalizes, through the new detail, the interest in 'those who build them' and thereby offers the opportunity to readjust the psychologically diminishing roles that all players in the design-to-build continuum have come to know. (DEAMER, 2010, p. 86-87).

A descrição do processo de produção, em 2003, da Porter House, do escritório Shop, em Nova Iorque, ilustra bem isso:

[...] we worked closely to the fabricators to understand the properties of the materials and the parameters that defined its manipulation. Each panel was laser-cut directly from our digital files and etched with a reference code that was keyed to installation drawings, which indicated location of panels, sequence of installation, and special instructions such as flashing details and mock-up requirements. While production of the panels was largely automated, technique was not eliminated - rather, it was incorporated into the details of the design. (Holden et al., 2012, p.131)

Questões ligadas à otimização do uso dos materiais, como o nesting dos polígonos a serem cortados, ou à sua montagem, como a gravação da numeração de cada peça, fazem parte de novas necessidades às quais o desenho do detalhe precisa atender. Sharples (2010) descreve outra obra do mesmo escritório, o edifício 290 Mulberry Street, em Nova Iorque, em que o detalhamento desenvolvido permitiu a viabilização econômica da fabricação dos painéis personalizados da fachada. A engenhosa solução consistiu na 
fresagem de uma base sobre a qual os tijolos puderam ser facilmente assentados, em ambiente industrial, conformando painéis pré-fabricados que depois foram içados nas laterais do edifício de dez pavimentos.

\section{PRODUÇÃO DIGITAL CONTEMPORÂNEA}

Nos últimos vinte anos, o rápido desenvolvimento e crescente disponibilidade de processos de fabricação digital e manufatura assistida por computador alteraram a lógica e a economia da produção industrial em série de elementos construtivos pré-fabricados. Menges (2013) descreve duas fases de evolução da fabricação digital na indústria da construção civil, ambas com impactos nos processos de projeto e de produção de arquitetura. A primeira caracterizou-se pela introdução de máquinas de controle numérico computacional (CNC), como fresadoras, serras e outras máquinas de marcenaria, que levaram a um aumento significativo da variabilidade nos processos de produção.

A utilização de equipamentos CNC torna o processo de fabricação muito mais flexível e, principalmente, garante um alto nível de precisão na execução de componentes construtivos. Isto se relaciona diretamente ao detalhamento arquitetônico, uma vez que, em um processo file-to-factory, o que é fabricado na indústria apresenta uma variação dimensional praticamente nula em comparação ao que foi projetado pelo arquiteto.

A fabricação dos componentes estruturais e de revestimento da fachada do Morpheus Hotel (Figura 1), projeto de Zaha Hadid Architects em Macau, é um bom exemplo do potencial de flexibilidade da produção, alta precisão dimensional e baixa incidência de erros. Segundo Salvi (2017), a variação dimensional das peças componentes dos nós, localizados na porção da fachada com curvatura complexa, foi de apenas dois milímetros.

Figura 1: Vista da Fachada do Hotel Morpheus, com as peças fabricadas instaladas. 


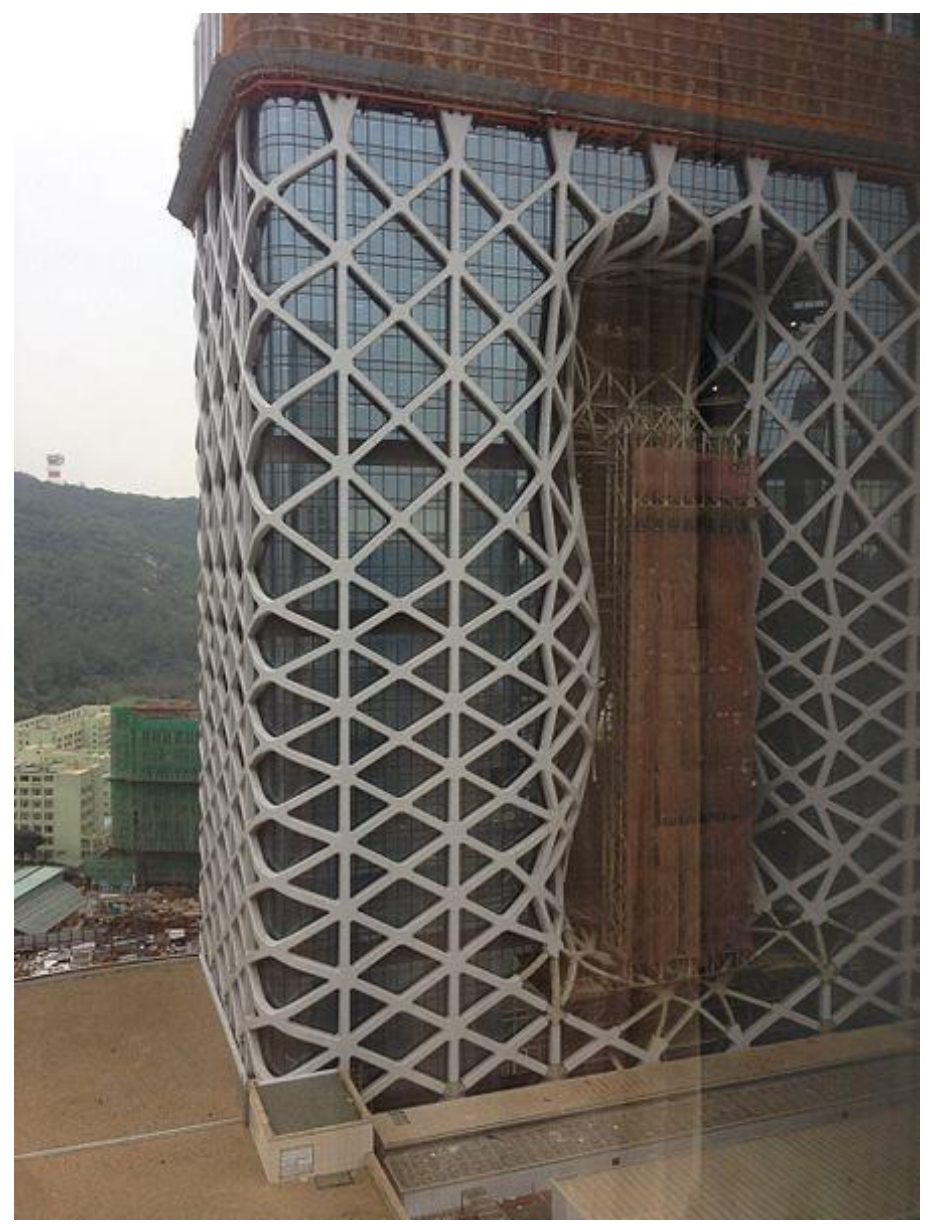

Fonte 1: DEVEREUX, Thom. Morpheus - City of Dreams: Construction 2017. 17 de janeiro de 2017. Disponível em: <https://commons.wikimedia.org/wiki/File:Morpheus__City_of_Dreams_(Macau)_-_Construction_2017.jpg >. Acesso em: 25 de outubro de 2017.

A segunda fase industrial da fabricação digital, conforme Menges (2013), está atualmente em implantação e é marcada por uma transição modal da produção, se deslocando do trabalho operacional de maquinário CNC para uma produção industrial assistida por robôs. Aponta-se alguns desafios que ainda precisam ser enfrentados na produção robótica: mesmo que os braços robóticos apresentem grande flexibilização do processo de produção, a relação da quantidade de seus eixos com o grau de precisão da produção é ainda inversamente proporcional. Desse modo, esta ainda não é a melhor opção quando se pretende alcançar um alto grau de precisão. Isto indica uma fase de exploração experimental de um equipamento que traz enorme potencial à produção industrial, principalmente por parte de pesquisadores e entusiastas.

Um exemplo de detalhamento arquitetônico produzido a partir do uso de fabricação robótica em um projeto exploratório é o The Framed Pavilion, do Institut für Architektur und Medien da TU-GRAZ.

Este projeto acadêmico de 2012 foi desenvolvido em duas etapas algorítmicas: a primeira se caracterizou pela definição das condições de contorno, projetando a forma geral da estrutura, e a segunda etapa determinou a elaboração de detalhes por encaixes com restrições construtivas e de 
fabricação, gerados por código robótico. Após a definição geral da estrutura, as barras foram organizadas em grupos de 10 unidades e dispostas lado a lado sobre uma estrutura de apoio inclinada para a realização da fresagem robótica, observando-se a incorporação de diferentes operações de usinagem e caminhos de abordagem do braço robótico (Figura 2). Em outras palavras, neste projeto o detalhamento dos encaixes só pode ser feito a partir do conhecimento do funcionamento do sistema robótico e de suas potencialidades e restrições.

Figura 2: Exemplo de fabricação robótica por usinagem.

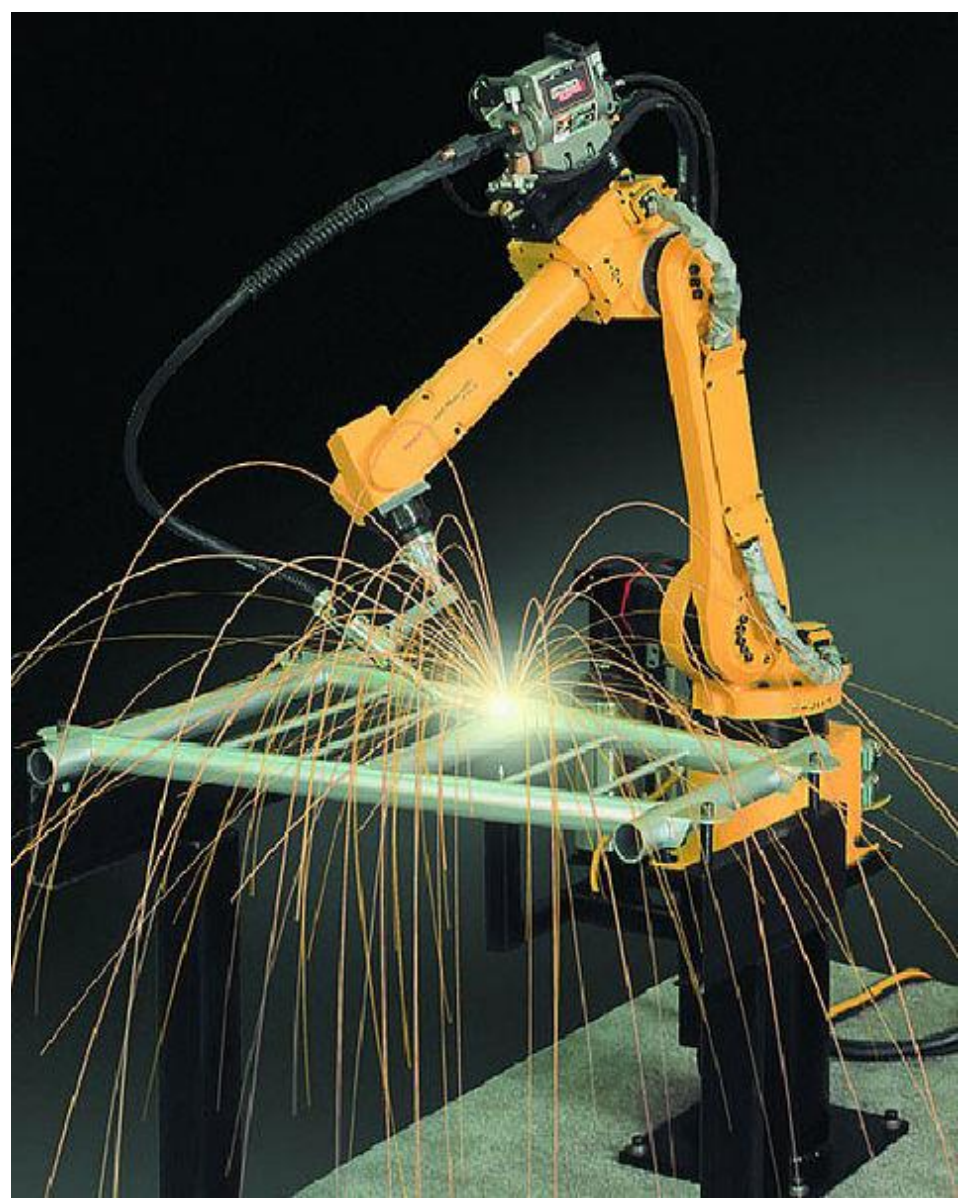

Fonte 2: FANUC ROBOTICS DEUTSCHLAND. Arc-Welding. 21 de dezembro de 2010.

Disponível em: <https://commons.wikimedia.org/wiki/File:Arc-welding.jpg>. Acesso em: 28 de outubro de 2017.

\section{BUILDING INFORMATION MODELING E A REPRESENTAÇÃO DO DETALHAMENTO ARQUITETÔNICO}

Outra questão muito importante na discussão sobre o futuro do detalhamento arquitetônico é sua representação digital. Com o uso dos sistemas BIM a representação do detalhe passa a se dar de maneira mais integrada com o desenvolvimento do projeto como um todo.

O objetivo do Building Information Modeling (BIM) é permitir que diferentes profissionais da área da construção possam colaborar 
simultaneamente no projeto. Nesse conceito, ao invés de se trabalhar em desenhos separados por disciplina, que poderiam conter contradições, trabalha-se em um único modelo compatibilizado, do qual são extraídas todas as informações, inclusive, pelo menos em tese, aquelas referentes aos detalhes. A visualização do modelo tridimensional do detalhe se tornaria mais dinâmica, e seria possível ampliá-lo quase que infinitamente na tela do computador. $\mathrm{Na}$ prática, contudo, sabe-se que, devido a restrições de hardware, isso nem sempre é possível.

Nas palavras de Eastman (2014, p.1) “[BIM] é um dos mais promissores desenvolvimentos na indústria relacionada à arquitetura, engenharia e construção (AEC)", ou, ainda, segundo Kassem e Amorim (2015, p.19): "trata-se de uma inovação tecnológica radical de processo, alterando funções, responsabilidades e conteúdos de produto ao longo de todo o ciclo de vida das construções". Deste modo, o detalhe no processo BIM ganha uma nova dimensão ao não se limitar à representação bidimensional. Torna-se um workflow para armazenamento e fluxo de informações desde pequenas escalas até à do edifício ao longo do projeto, construção e demolição.

Neste contexto, o LOD de um modelo em BIM é um conceito que tem como objetivo garantir a legibilidade da representação, adequando a quantidade de informações ao grau de desenvolvimento do projeto e à escala da visualização desejada. O BIMForum (2016) destaca a diferença entre Level of Development e Level of Detail. 0 último diz respeito à quantidade de detalhes associada à geometria, enquanto o primeiro refere-se ao grau de complexidade na qual a geometria e suas informações se encontram.

O grupo Vico Software e Webcor Builders iniciaram estudos a respeito do nível de detalhe com o Model Progression Specification (MPS), em 2004 como coloca Bedrick, (2008). Em 2008, o The American Institute of Architects continuou tais estudos por meio do protocolo E202, formalizando a criação do LOD. Três anos depois, o BIMForum firmou o desenvolvimento de especificações, formando o primeiro grupo de trabalho de sistematização e extensa exemplificação do LOD aplicado em sistemas construtivos.

O sistema LOD está dividido em seis classificações, de 100 a 500, progredindo de um nível mais conceitual de modelo até outro de maior complexidade de informação, onde cada um dos níveis engloba o anterior. 0 LOD é independente da escala e está vinculado tanto ao componente quanto ao todo. Por exemplo, um elemento construtivo pode estar modelado em LOD 200, mas associado a modelos e/ou informações em LOD 100, e vice-e-versa. Tudo depende da demanda e finalidade do modelo. Tanto o BIMForum (2016) como Morais, Granja e Ruschel (2015) trazem possíveis definições para os produtos esperados em cada uma das classificações LOD.

O LOD 100, conceptual, é o nível mais baixo de classificação. Não são representações geométricas precisas, mas uma aproximação quanto a forma, localização e tamanho. 0 componente pode até ser representado por meio de símbolos que mostrem a sua existência. Morais, Granja e Ruschel (2015), ao tratar da escala do edifício, afirmam que este nível é próprio para estudos 
conceituais iniciais, como é o caso de estudos volumétricos que permitam o entendimento das relações de implantação, orientação ou de custo por $\mathrm{m}^{2}$.

Em seguida há o LOD 200, approximate geometry, onde objetos, sistemas, forma, dados quantitativos, localização e orientação são aproximados. Conjuntos de informações não gráficas podem ser apresentados com maior refinamento que o LOD 100. Quando se fala da escala do edifício, este nível é equivalente a um anteprojeto. Parte dos sistemas prediais deve ser resolvida. Nesta etapa, já podem ocorrer as primeiras simulações de desempenho e extração de dados quantitativos.

Em sequência, chega-se ao LOD 300 com uma geometria precisa, precise geometry. 0 modelo expressa as informações exatas de quantidades, tamanho, forma, localização e orientação. Logo, as informações, como dimensões, podem ser extraídas diretamente do modelo sem a necessidade de informações não modeladas anexas. Nesta fase o projeto está quase definido pois as especificações de materiais e dados quantitativos já permitem a documentação para a obra, lista de compras e cronograma de obra, mesmo que em linhas gerais.

A quarta fase é o LOD 350, assemblies for coordination, um nível de desenvolvimento no qual são modeladas as partes necessárias para coordenação e compatibilização entre os diferentes sistemas. São modelados suportes e conexões ou outros detalhes que interfiram na sua compatibilização.

O próximo estágio é o LOD 400, fabrication, que apresenta detalhes em precisão suficientes para a fabricação do componente ou construção do edifício. Assim, o modelo carrega todas as informações necessárias, não necessitando de informações não modeladas. Dada essa precisão, esta etapa permite análises de desempenho e eficiência precisas.

Por último, há o as built como LOD 500, ou seja, ocorre depois que o edifício é construído a fim do controle de sua operação, manutenção e reforma. A Figura 3 exemplifica graficamente as definições supracitadas.

Figura 3: Exemplo de definição LOD para pilar metálico.

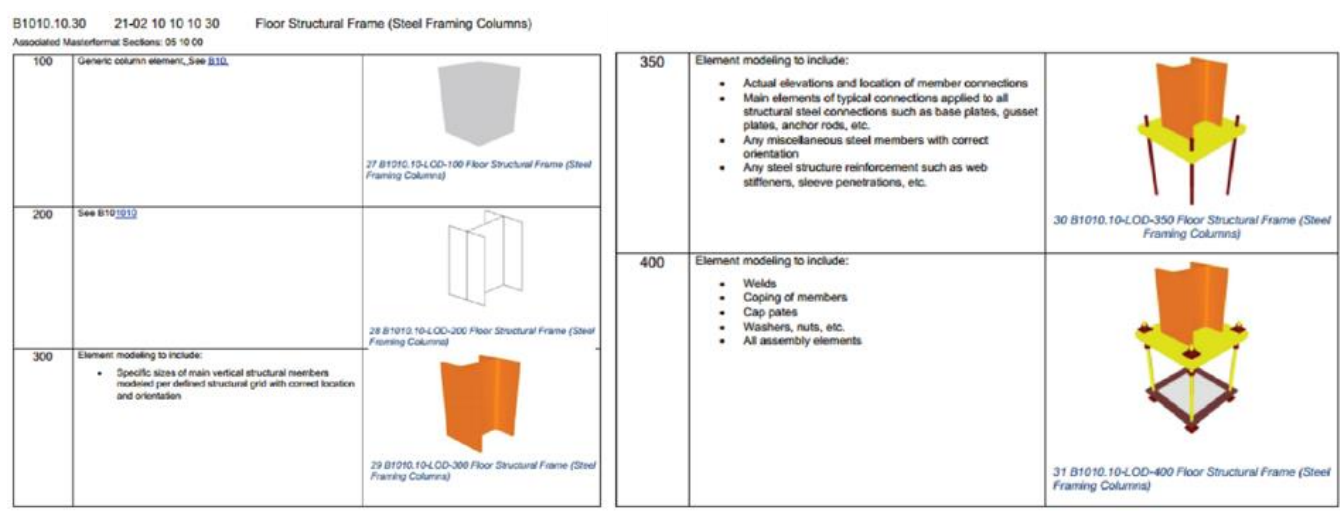

Fonte 2: BIMForum, 2016, p. 42. 
O LOD possibilita que os diferentes profissionais na área de construção estabeleçam padrões de demanda e produto detalhados e, consequentemente, de desenvolvimento, o que melhora significativamente a eficiência do fluxo de informações (Figura 4). Nas etapas iniciais, devido ao LOD ser mais baixo, o modelo é mais genérico e flexível. Ao longo do desenvolvimento do projeto, o LOD aumenta e o modelo se aproxima do que seria esperado de um As Built como descrevem Fai e Rafeiro (2014). Dessa forma, é possível compreender o BIM como processo de projeto, bem como uma ferramenta para o ensino de detalhamento e detalhe na formação de arquitetos e engenheiros, pois permite uma visão holística do processo construtivo e do detalhe. Ao contrário dos desenhos 2D e 3D baseados em entidades (e não em elementos construtivos) "[...] o processo BIM melhora sensivelmente a visualização espacial do que está sendo concebido. [...] Assim, os desenhos abstratos, sem quaisquer vínculos com a realidade da construção, são substituídos por desenhos precisos e detalhados" (Flório, 2007. p.7).

Figura 4: Projeto de restauro do edifício West Block do Parliament Hill em Ottawa, Canadá, realizado por Carleton Immersive Media Studio (CIMS), um exemplo da aplicação do BIM no desenvolvimento de detalhes arquitetônicos, com três níveis de detalhamento: elevações de uma abertura em arco duplo encontrado no West Block. A-E expressam a progressão do modelo iniciado com a (A) fotogrametria, (B) desenho CAD, (C) LOD 1, (D) LOD 2 e (E) LOD 3.

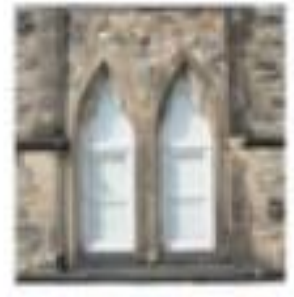

A

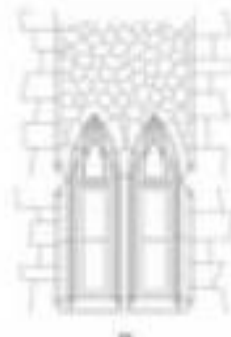

s

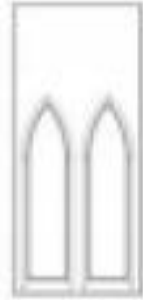

e

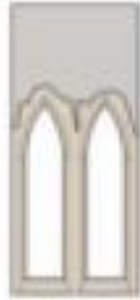

a

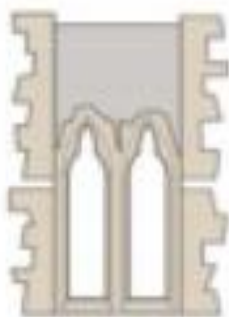

i

Fonte 3: FAI, S.; RAFEIRO, J. 2014, p. 127.

Ao contrário do que ocorreu na área de engenharia mecânica, na arquitetura o uso de sistemas CAD e, mais recentemente, de sistemas BIM, foi implementado antes da popularização do emprego de sistemas CAM para a construção civil. Como resultado, os sistemas BIM nem sempre estão plenamente integrados com os desenhos complementares de produção digital, como os desenhos de planificação de superfície ou de nesting, ou ainda com a exportação de arquivos para a operação direta de equipamentos CNC, fazendose necessário ainda, o uso de representações específicas para essas finalidades. Uma das raras exceções é o software Generative Components, da Bentley, que pode ser integrado à plataforma BIM Microstation, e possui ferramentas específicas de planificação e exportação de G-code, como exposto por Aish (2017). Além disso, a integração entre sistemas BIM e desenhos para fabricação digital pode ser obtida por meio de programação e do uso de addons com os plug-ins Dynamo e o Grasshopper 3D, para as plataformas BIM, no primeiro caso para Revit e no segundo para Archicad e VisualArq, por exemplo. 
Outra importante questão que tem surgido no desenvolvimento de detalhes arquitetônicos é o uso da modelagem paramétrica e a geração algorítmica de detalhes. Esses recursos se fazem necessários quando a variabilidade dos detalhes em uma obra é muito grande. Nesses casos, podese inserir variações paramétricas do detalhe em cada ponto específico do projeto, ou então os detalhes podem ser gerados automaticamente por programação, ao invés de serem modelados diretamente. Esse foi o método utilizado no desenvolvimento do projeto do edifício 30 St. Mary Axe em Londres, projetado pelo escritório de Norman Foster e inaugurado em 2004, também conhecido como The Gerkin. Segundo Hugh Whitehead, do Specialist Modeling Group do escritório de Foster, como relata Menges (2010, p. 30): "the Swiss Re building that forced us to address the problem of how to design and produce details that are programmed rather than drawn. At each floor, the rules are always the same, but the results are always different".

\section{DISCUSSÃO E IMPACTOS PARA O ENSINO}

0 detalhamento do edifício sempre teve um papel importante na viabilização técnica da edificação. Se durante o período de industrialização em massa ele se apoiava em soluções prontas, predefinidas por uma equipe de desenhistas industriais, na era da produção pós-industrial as possibilidades são muito mais amplas e ele depende agora do conhecimento sobre as técnicas de fabricação por parte do projetista.

Com o crescimento exponencial do poder computacional a profissão do arquiteto entrou em uma nova fase, marcada por tecnologias como as simulações computacionais, o BIM, o CAM e a produção robótica. Esta nova fase da produção arquitetônica, segundo Hauck, Bergin e Bernstein (2017), demanda habilidades digitais transdisciplinares aplicadas ao projeto e à construção, assim como o projeto colaborativo interdisciplinar e integrado e o realinhamento das profissões de arquiteto e engenheiro.

A relação entre o detalhe e a fabricação digital torna necessária não apenas uma revisão, mas a proposição de uma nova abordagem conceitual, que poderia ser definida como Detalhe de Precisão. Uma das questões envolvidas nessa nova abordagem é o risco do desenvolvimento do detalhe no ambiente BIM. Assim como em outras ferramentas de desenho e modelagem digital, há a tentação de se utilizar apenas elementos padronizados e já disponibilizados como "famílias" ou "templates". O ambiente em si, por conter toda a complexidade do projeto em forma de módulo geométrico tridimensional, tem a vantagem de mostrar o local em que o detalhe deve ser implantado com elevado grau de informações contextuais, mas ao mesmo tempo pode impedir o nível de abstração necessário ao processo criativo. A fim de evitar tal alienação, é indispensável o ensino e exercício do detalhe para que arquitetos e engenheiros tenham independência no processo de projeto e autonomia diante dos desafios construtivos que surgem com o exercício da profissão.

Outra importante questão é a incorporação do projeto algorítmico e da fabricação digital na formação dos arquitetos. Os arquitetos formados 
atualmente em algumas instituições adquirem extenso conhecimento em robótica e fabricação digital, aplicando-os tanto no desenvolvimento de pesquisas acadêmicas quanto em inovadoras práticas profissionais de arquitetura. 0 National Centre for Competence in Research (NCCR) for Digital Fabrication, DFAB na ETH Zurich, Suíça, é uma das instituições de ensino e pesquisa que exemplificam essa abordagem, com a meta de ensinar objetivamente os alunos a projetarem de forma algorítmica, a fim de alcançarem o controle de todas as etapas da produção.

Para capacitar os arquitetos dessa geração a elaborarem detalhes condizentes com os novos meios de produção será necessário, em breve, formalizar uma teoria do detalhamento arquitetônico para o século XXI - e de sua representação - do mesmo modo que os livros de Ching, Allen e Rand e Deplazes formalizaram o conhecimento sobre os métodos construtivos da arquitetura do século XX e a representação de seu detalhamento. Um livro com esse objetivo precisará reunir elementos sobre fabricação e montagem, como na obra Product Design for Manufacture and Assembly de Boothroyd, Dewhurst e Knight (2002), além de inúmeros exemplos de detalhes de construções representados parametricamente ou algoritmicamente e produzidos com técnicas de fabricação digital. Mas o fato dessa obra ainda não existir não significa que não devamos começar desde já a refletir sobre as necessidades de transformação da formação de nossos arquitetos no que se refere à concepção e à representação do detalhamento arquitetônico.

\section{REFERÊNCIAS}

ABNT - ASSOCIAÇÃO BRASILEIRA DE NORMAS TÉCNICAS. NBR 6492 Representação de Projetos de Arquitetura. Rio de Janeiro: ABNT, 1994.

AISH, R. Bentley's Generative Components: A design tool for exploratory architecture. Disponível em: <http://195.250.185.245:8080/MyWeb/get/Bentley_training/GenerativeCo mponents.pdf>. Acesso em: 29 de setembro de 2017.

ALLEN, E.; RAND, P. Architecture Detailing: Function, Constructibility, Aesthetics. Wiley, 2 ed, 2007. 384p.

ASBEA - Associação dos Escritórios de Arquitetura. Detalhes em Arquitetura. São Paulo: J. J. Carol, 2010.

BEDRICK, Jim. Organizing the Development of a Building Information Model. In: AECbytes, Agosto, 2008. Disponível em: <http://www.aecpe.com/08-0820\%20MPS.pdf>. Acesso em: 10 de outubro de 2017.

BIMFORUM. Level of Development Specification - Version 2016. Issued: Oct. 19, 2016 
BoothroyD, G.; DEWhURST, P.; KNIGHT, W. A. Product Design for Manufacture and Assembly. Nova Iorque: Marcel Dekker, 2002.

CELANI, G.; URANO, R. Do protótipo ao prototipado: personalização em massa versus utopias do século XX na arquitetura e urbanismo. In: PARC Pesquisa em Arquitetura e Construção. v. 07, n. 03. p. 160 - 169. Campinas, 2016. Disponível em: <https://periodicos.sbu.unicamp.br/ojs/index.php/parc/article/view/8647 348>. Acesso em: 10 de outubro de 2017.

COSTA, A. F. Detalhando a Arquitetura 11. Rio de Janeiro: Do autor, 1997. $167 \mathrm{p}$.

DEAMER, P. Detail deliberations (originalmente publicado em 2008). In: BERNSTEIN, P.; DEAMER, P. (eds). Building (in) the Future: Recasting Labor in Architecture. Nova Iorque: Princeton Architectural Press, 2010, p.81-88.

DEPLAZES, Andrea. Constructing Architecture: Material, Processes, Structures. Suíça, Birkhäuser Architecture, 2005. 479 p.

EASTMAN, C.; LISTON, K.; SACKS, R.; TEICHOLZ, P. Manual de BIM: um guia de modelagem da informação da construção para arquitetos, engenheiros, gerentes, construtores e incorporadores. Porto Alegre: Bookman, 2014. 483 p.

FAI, S.; RAFEIRO, J. Estabilishing an appropriate level of detail (LOD) for a Building Information Model (BIM) - West Block, Parliament Hill, Otawwa, Canada. In: ISPRS Annals of the Photogrammetry, Remote Sensing and Spatial Information Sciences, Volume II-5, 2014. ISPRS Technical Commision V Symposium, 23 - 25 June, 2014, Riva del Garda, Italy.

FLÓRIO, W. Tecnologia da informação na construção civil: contribuições do Building Information Modeling no processo de projeto em arquitetura. In: III Forúm de Pesquisa FAU Mackenzie. São Paulo, 2007. Disponível em: <http://www.mackenzie.com.br/fileadmin/Graduacao/FAU/Publicacoes/PD F_IIIForum_b/MACK_III_FORUM_WILSON_FLORIO.pdf>. Acesso em: $07 \mathrm{de}$ setembro de 2017.

FORD, Edward. The Architectural Detail. United States: Princeton Architectural Press, 2011. 336 p.

FORD, Edward. The Grand Work of Fiction: The Detail as Narrative. In: Architecture Design: Future Details of Architecture. Wiley, v. 84, n. 04, p. 26 - 35, jul./ago. 2014.

FOLHA DE SÃO PAULO. Ranking Universitário Folha 2016. Disponível em: $<$ http://ruf.folha.uol.com.br/2016/ranking-de-cursos/arquitetura-eurbanismo/>. Acesso em 20 de agosto de 2017. 
GARCIA, Mark. Histories, Theories and Futures of the Details of Architecture. In: Architecture Design: Future Details of Architecture. Wiley, v. 84, n. 04, p. 14 - 25, jul./ago. 2014.

GROPIUS, Walter. Um vigoroso movimento. In: XAVIER, Alberto (org.). Depoimento de uma geração: Arquitetura Moderna Brasileira. São Paulo, Cosac Naify, 2003. p. 153 - 154.

HAUCK, A.; BERGIN, M.; BERNSTEIN, P. The Triumph of the Turnip. In: Fabricate 2017 - Rethinking Design and Construction. p. 16 - 21. Londres: UCL Press, 2017.

HOLDEN, K.; PASQUARELLI, G.; SHARPLES, C.; SHARPLES, C. D.; SHARPLES, W. SHoP: Out of Practice: Nova Iorque: Monacelli Press, 2012. 51 p.

IAB - INSTITUTO DE ARQUITETOS DO BRASIL. Roteiro para o desenvolvimento do projeto de arquitetura da edificação. 7 p. Disponível em: <http://www.iab.org.br/sites/default/files/documentos/roteiroarquitetonico.pdf >. Acesso em: 15 de setembro de 2017.

KASSEM, M.; AMORIM, S. R. L. BIM - Building Information Modeling no Brasil e na União Europeia: Diálogos Setoriais União Europeia - Brasil. Brasília: Ministério do Planejamento, Orçamento e Gestão, 2015. 159 p. Disponível em: <http://sectordialogues.org/sites/default/files/acoes/documentos/bim.pdf >. Acesso em: 27 de setembro de 2017.

KOLAREVIC, Branko. Why we need architecture of Tolerance. In: Architecture Design: High Definition - Zero Tolerance in Design and Production. Wiley, v. 84, n. 01, p. 128 - 132, jan./feb. 2014.

LOOS, Adolf. Ornament and Crime (originalmente publicado em 1908). In: MITCHELL, Michael (trad.). Adolf Loos - Ornament and Crime: Selected Essays. Ariadne Press, 1997. 206 p.

MENGES, A. Instrumental Geometry. In: CORSER, R. (ed.). Fabricating Architecture: Selected Readings in Digital Design and Manufacturing. Princeton Press, New York, 2010, 216p.

MENGES, A. Morphospaces of Robotic Fabrication: From theoretical morphology to design computation and digital fabrication in architecture. In: RobArch 2012 - Robots in Architecture, Arts and Design Conference. Springer-Verlag, Viena - Áustria, 2013.

MORAIS, M.; GRANJA, A. D.; RUSCHEL, R. C. Restrições orçamentárias e entrega de valor: sinergias entre BIM e custeio-meta. In: Gestão e Tecnologia de Projetos, São Paulo, v. 10, n. 1, p. 7-27, jan./jun. 2015. Disponível em: 
<http://dx.doi.org/10.11606/gtp.v10i1.81141>. Acesso em 23 de agosto de 2017.

NOLTE, Tobias. WITT, Andrew. Gehry Partners' Fondation Louis Vuitton: Crowdsourcing Embedded Intelligence. In: Architecture Design: High Definition - Zero Tolerance in Design and Production. Wiley, v. 84, n. 01, p. 82 - 89, jan./feb. 2014.

OXMAN, Rivka; OXMAN, Robert. The New Structuralism: Design, Engineering and Architectural Technologies. Academy Press, 2010. 136 p.

QS TOPUNIVERSITIES. Top Architecture Schools in 2016. Disponível em: $<$ https://www.topuniversities.com/university-rankings-articles/universitysubject-rankings/top-architecture-schools-2016>. Acesso em: 20 de agosto de 2017.

SALVI, Michele. The Morpheus Hotel: From Design to Production. In: Live Webinar. Disponível em: <https://vimeo.com/203509846>. Acesso em 11 de setembro de 2017.

SHARPLES, C. D. Technology and labor. In: BERNSTEIN, P. DEAMER, P. (eds). Building (in) the Future: Recasting Labor in Architecture. Nova Iorque: Princeton Architectural Press, 2010, p.91-99.

SILVA, N. F.; BRIDGES, A. H.; LIMA, E. M.; MORAIS, H. R. A.; JÚNIOR, F. A. S. A indústria da construção civil está pronta para a fabricação digital e a customização em massa? Uma pesquisa sobre um caso Brasileiro. In: Sigradi 2009, São Paulo, 2009. Disponível em:<http://lecomp.fau.unb.br/moodle/file.php/26/sigradi2009/1079_Nean der_Furtado_Silva_Sigradi9.pdf $>$. Acesso em: 31 de agosto de 2017.

VASCONCELOS, A. C. O Concreto no Brasil: pré-fabricação, monumentos, fundações. Volume III. Studio Nobel. São Paulo, 2002. 